\title{
Estudios de las condiciones óptimas para maximizar estabilidad física y oxidativa de un ingrediente vitamínico nanoencapsulado*
}

\section{Studies of optimal conditions to maximize physical and oxidative stability of nanoencapsulated vitamin ingredient}

\author{
Mujica-Álvarez, J., ${ }^{* *}$ Matiacevich, S., ${ }^{* * *}$ y Bustos, R. ${ }^{* *}$
}

\begin{abstract}
The aim of this work was to evaluate the effect of different wall materials, sodium caseinate (CS) and a modified starch Capsul ${ }^{\circledR}$ (CAP), on the physical and oxidative stability of nanoemulsions with vitamin $\mathrm{E}$, to obtain a nanoencapsulated vitamin ingredient. An experimental Box-Behnken design was established, where three factors were analyzed: type of encapsulating agent ( $4 \% \mathrm{w} / \mathrm{w}$ CS/CAP/CAP+CS), surfactant concentration Tween $80(1-2 \% \mathrm{w} / \mathrm{w})$, and ultrasound time (1-3 $\mathrm{min}$ ). The response variables were the size of nanocapsules, polydispersity index (PDI) and oxidative stability at 80,110 and $140{ }^{\circ} \mathrm{C}$, expressed as the induction period (PI). From the optimal conditions, the model was validated and the concentration of the encapsulating agent was increased to improve the physical stability of the nanoemulsion during storage $\left(4^{\circ} \mathrm{C}-72 \mathrm{~h}\right)$. These nanoemulsions were lyophilized and the PI was evaluated in comparison with free vitamin $E$ and as a nanoemulsion. A particle size between 20-100 $\mathrm{nm}$ was obtained with a $\mathrm{PDI}<0.5$ under all experimental conditions, confirming the obtaining of nanoemulsions with a monomodal size distribution. The lyophilization process improved the oxidative stability of the vitamin compared to the vitamin $\mathrm{E}$ nanoemulsion and the free vitamin $\mathrm{E}$.
\end{abstract}

KEYWORDS: encapsulation, $\alpha$-tocopherol, nanoemulsion, oxidative stability, physical stability, lyophilization.

RESUMEN: El presente trabajo tuvo como objetivo evaluar el efecto de distintos materiales de pared, caseinato de sodio (CS) y un almidón modificado Capsul ${ }^{\circledR}(\mathrm{CAP})$, sobre la estabilidad física y oxidativa de nanoemulsiones con vitamina $E$, para obtener un ingrediente vitamínico nanoencapsulado. Se estableció un diseño experimental Box-Behnken, en donde se analizaron tres factores: tipo de agente encapsulante $(4 \% \mathrm{p} / \mathrm{p} \mathrm{CS} / \mathrm{CAP} / \mathrm{CAP}+\mathrm{CS})$, concentración de surfactante

Recibido: 4 de noviembre de 2018.

Aceptado: 18 de diciembre de 2018.

* Este trabajo de investigación fue financiado por el proyecto CEN-LEITAT-USACH, CORFO 13CEI2-21839. Se agradece, además, la participación y colaboración del Proyecto Fortalecimiento USA1799_MS172228 del Departamento de Ciencia y Tecnología de los Alimentos de la Universidad de Santiago de Chile.

** Universidad de Santiago de Chile, Facultad de Ingeniería, Departamento de Ingeniería Química. Av. Libertador Bernardo O'Higgins 3363, Estación Central, Región Metropolitana, Chile.

*** Universidad de Santiago de Chile, Facultad Tecnológica, Departamento de Ciencia y Tecnología de los Alimentos.

Autora de correspondencia: silvia.matiacevich@usach.cl 
Tween 80 (1-2\% p/p), y tiempo de ultrasonido (1-3 min). Las variables de respuesta fueron el tamaño de nanocápsulas, índice de polidispersidad (PDI) y estabilidad oxidativa a 80,110 y $140{ }^{\circ} \mathrm{C}$, expresados como periodo de inducción (PI). A partir de las condiciones óptimas, se validó el modelo y se aumentó la concentración del agente encapsulante para mejorar la estabilidad física de las nanoemulsiones durante su almacenamiento $\left(4^{\circ} \mathrm{C}-72 \mathrm{~h}\right)$. Estas nanoemulsiones fueron liofilizadas y se les evaluó el PI en comparación con la vitamina E libre y en nanoemulsión. Se obtuvo un tamaño de partícula entre $20-100 \mathrm{~nm}$ con un PDI<0.5 en todas las condiciones experimentales, confirmándose la obtención de nanoemulsiones con una distribución de tamaño monomodal. El proceso de liofilización mejoró la estabilidad oxidativa de la vitamina en comparación con la nanoemulsión de vitamina E y la vitamina E libre.

PALABRAS CLAVE: encapsulación, $\alpha$-tocoferol, nanoemulsión, estabilidad oxidativa, estabilidad física, liofilización.

\section{Introducción}

La encapsulación es una tecnología ampliamente utilizada en la industria alimentaria para la protección de compuestos bioactivos que son sensibles a la luz, oxígeno, humedad, calor y otros agentes ambientales (Katouzian y Jafari, 2016). La emulsificación (aceite en agua) es una metodología de encapsulación donde un compuesto lipofílico de interés es protegido por interacción con un surfactante y/o agente encapsulante o material de pared, permitiendo preservarlo en el tiempo, especialmente cuando debe incorporarse en una matriz alimentaria (McClements, 2016; Ghani et al., 2017). Particularmente, dentro de los compuestos de interés se encuentran las vitaminas lipofílicas como la vitamina E, pues posee propiedades antioxidantes que tienen un papel clave en el sistema inmune y procesos metabólicos $(\mathrm{NIH}$, 2018). La encapsulación de vitaminas lipofílicas presenta ventajas tales como proteger el compuesto de factores ambientales, reducir su interacción con otras vitaminas y/o moléculas presentes en un alimento, mejorar su estabilidad y biodisponibilidad (por ejemplo, mediante la disminución del tamaño de gota), y liberación (Dasgupta y Ranjan, 2018; Katouzian y Jafari, 2016). Para ello, una de las técnicas de encapsulación más utilizada es la nano-emulsificación (tamaños de gotas $<100 \mathrm{~nm}$ ), donde los parámetros críticos en su estabilidad física son el tipo de agente encapsulante/surfactante utilizado, así como las condiciones de proceso para su formación mediante técnicas de alta energía, como la homogenización por ultrasonido. Este último genera la formación y el colapso de las gotas presentes en una emulsión mediante el fenómeno de cavitación acústica, dando origen a las nanoemulsiones (Leong et al., 2016).

Dentro de los agentes encapsulantes más utilizados para compuestos lipofílicos están las proteínas y algunos almidones modificados. Esto por sus propiedades anfipáticas y/o emulsificantes. De estos compuestos destacan el caseinato de sodio, debido a su alta solubilidad y capacidad de homogenizarse en presencia de grasas o aceites (Rubio-Anaya y Guerrero-Beltrán, 2012), y el almidón modificado Capsul ${ }^{\circledR}$, el cual presenta excelentes propie- 
dades emulsificantes, baja viscosidad y peso molecular, y actúa como barrera frente al oxígeno (Quirós-Sauceda et al., 2014).

Si bien algunos materiales de pared poseen zonas tanto hidrofílicas e hidrofóbicas, es común la adición de agentes surfactantes para mejorar la formación y estabilidad de la emulsión. Estos corresponden a sustancias tensoactivas anfipáticas que normalmente se posicionan en la interfase compuesto activo-material de pared. También pueden actuar como emulsificantes. Sin embargo, su uso debe ser controlado ya que puede alterar las propiedades de la emulsión mediante la interacción con el material de pared o formación de micelas del mismo (McClements, 2016).

Para almacenar emulsiones y mejorar sus propiedades, se utiliza comúnmente la técnica de secado, obteniéndose así sólidos encapsulados y estables tanto física como microbiológicamente (Vidya et al, 2015). Uno de los métodos para secado de emulsiones que en los últimos años ha ido ganando terreno es el proceso de liofilización, el cual se basa en la sublimación del disolvente y se divide en tres etapas: congelación, secado primario (etapa de sublimación) y secado secundario (etapa de desorción), obteniéndose así un producto final en formato polvo (Gómez-Cruz y Jiménez-Munguía, 2014).

La técnica de liofilización presenta un costo mayor en comparación con otros procesos, como, por ejemplo, el secado por atomización, pues demanda un alto consumo de energía y largo tiempo de procesamiento. Sin embargo, se ha probado que la liofilización permite eliminar el agua de nanoemulsiones y formar nanocápsulas manteniendo íntegra tanto su forma como su estructura (Gómez-Cruz y Jiménez-Munguía, 2014). Una gran ventaja de esta técnica es que sustancias que son susceptibles a oxidarse, como las vitaminas, quedan protegidas bajo condiciones de vacío durante el secado y pueden conservarse por largos periodos al removerse entre el 95 y el 99\% del agua. Adicionalmente, existe baja o nula pérdida de sustancias químicas volátiles y los nutrientes sensibles al calor no se ven afectados por el proceso de secado (Nireesha et al., 2013).

A partir de los antecedentes mencionados, el trabajo que se presenta a continuación tuvo como objetivo evaluar el efecto del uso de distintos materiales de pared (una proteína: caseinato de sodio, y un almidón modificado: Capsul ${ }^{\circledR}$ ) sobre el tamaño de partícula, y la estabilidad física y oxidativa de nanoemulsiones con vitamina E (VE), para así obtener un ingrediente vitamínico en formato polvo que pueda ser incorporado en una matriz alimentaria.

\section{Procedimiento experimental}

\section{Materiales}

La vitamina E (DL- $\alpha$-Tocopherol) y el surfactante no iónico Tween 80 fueron comprados en Sigma Aldrich (EEUU). Los agentes encapsulantes, caseinato de sodio (CS) y el almidón modificado Capsul ${ }^{\circledR}$ (CAP), fueron donados por Blumos (Chile) y Quimatic (Chile), respectivamente. 


\section{Preparación de nanoemulsiones}

La fase acuosa $(88 \%$ p/p) se preparó mediante la suspensión de los agentes encapsulantes (CS o CAP) en agua destilada según diseño experimental. Esta fase se agitó magnéticamente a $650 \mathrm{rpm}$ a $65^{\circ} \mathrm{C}$ durante $2 \mathrm{~h}$. Luego, la fase oleosa $(12 \% \mathrm{p} / \mathrm{p})$, compuesta por la vitamina E (11\% p/p) y Tween $80(1 \%$ $\mathrm{p} / \mathrm{p})$, se incorporó gota a gota dentro de la fase acuosa, agitándose en un homogeneizador Ultra-Turrax (TR50. Trhistor, Germany) operado a 12,400 rpm durante $5 \mathrm{~min}$. Finalmente, se obtuvieron nanoemulsiones a través de un tratamiento de homogenización por ultrasonido (QSonica, USA) a $80 \%$ de amplitud con un poder de densidad de $400 \mathrm{~W} / \mathrm{L}$ a diferentes tiempos. La nanoemulsión final se secó en un equipo liofilizador IlShin FD550B (Ilshin, Corea) a $-50^{\circ} \mathrm{C}$ y 5 mTorr durante $48 \mathrm{~h}$.

\section{Diseño experimental}

Se estableció un diseño Box-Behnken para optimizar los parámetros de formulación de las nanoemulsiones, utilizando el programa Statgraphics Centurion XVI.I. Se evaluaron tres variables independientes: tipo de encapsulante (AE): caseinato de sodio $4 \% \mathrm{p} / \mathrm{p}$, Capsul ${ }^{\circledR} 4 \% \mathrm{p} / \mathrm{p}$ y una mezcla $50 / 50$ de ambos; concentración de Tween $80(1 ; 1,5 ; 2 \%$ p/p) y tiempo de ultrasonido (1-3 min). Las variables dependientes fueron: tamaño de partícula, índice de polidispersidad (PDI) y estabilidad oxidativa. Se realizó una optimización de múltiple respuesta utilizando la función de deseabilidad, minimizando el tamaño de partícula y PDI, y maximizando los periodos de inducción de la estabilidad oxidativa.

\section{Caracterización de nanoemulsiones}

Tamaño promedio de partícula e índice de polidispersidad

Se determinaron por dispersión de luz dinámica en un equipo Zetasizer Nano $S$ (Malvern Instruments, GB) a $25^{\circ} \mathrm{C}$. Todas las mediciones se realizaron por duplicado de 12 lecturas cada una.

\section{Análisis de estabilidad oxidativa}

Se realizó en un equipo RapidOxy (Anton Paar, Alemania) a 80, 110 y 140 ${ }^{\circ} \mathrm{C}$ en atmósfera oxidante. La muestra se introduce en una cámara y una vez sellada esta se carga con oxígeno, hasta una presión fija de $700 \mathrm{kPa}$, y se calienta hasta la temperatura de análisis. El consumo de oxígeno es marcado por una disminución de la presión dentro de la cámara. La medición es continua hasta el punto de ruptura, el cual corresponde a una caída de presión definida equivalente al $10 \%$. El resultado se reporta como el periodo de inducción (PI), el cual corresponde al tiempo transcurrido entre el inicio de la prueba y el punto de ruptura $\left(\triangle \mathrm{P}_{\mathrm{O} 2} 10 \%\right)$, indicando la estabilidad de la muestra frente a la oxidación. De esta manera, si el PI aumenta, la estabilidad oxidativa de la muestra es mayor y tardará más tiempo en oxidarse. 
Las temperaturas seleccionadas para el análisis se eligieron con base en las utilizadas en procesos industriales durante la producción y procesamiento de alimentos (por caso, extrusión).

\section{Análisis de estabilidad fisica}

El grado de estabilidad física de las nanoemulsiones se determinó utilizando un equipo Turbiscan (Formulaction, Francia) a una temperatura de almacenamiento de las muestras a $4{ }^{\circ} \mathrm{C}$.

\section{Análisis estadístico}

Las mediciones se realizaron por triplicado y los resultados se reportaron como valores promedio con su respectiva desviación estándar. El análisis estadístico de los resultados obtenidos se evaluó mediante ANOVA con un nivel de significancia del 0.05 ( $p<0.05$ ) y un intervalo de confianza del 95\%. Al existir una diferencia estadísticamente significativa entre las medias se realizó el test de Tukey HSD para la comparación de las muestras. Todos los análisis estadísticos se realizaron en el software GraphPad Prism v7 (GraphPad Software, CA., EEUU).

\section{Resultados y discusión}

\section{Tamaño y distribución de partícula de las nanoemulsiones con vitamina $E$}

La tabla 1 muestra los resultados de tamaño promedio de nanopartícula de las 15 corridas experimentales del diseño experimental Box-Behnken. Este diseño corresponde a un tipo de diseño de superficie de respuesta. Particularmente, en este estudio se evaluaron 3 factores de 2 niveles cada uno, correspondientes a 12 corridas experimentales más 3 puntos centrales, dando así un total de 15 corridas. Se obtuvo un tamaño de partícula entre los 20 y $100 \mathrm{~nm}$ en todas las condiciones experimentales, confirmándose la obtención de nanoemulsiones. Las nanoemulsiones que presentaron menor tamaño fueron aquellas cuyo tipo de agente encapsulante fue el caseinato de sodio y Tween 80 a una alta concentración $(1.5 \% \mathrm{p} / \mathrm{p})$. Por otro lado, se determinó el índice de polidispersidad, el cual corresponde a un valor que va desde 0 a 1 e indica heterogeneidad en la distribución de tamaño de nanopartícula de las muestras, los valores más cercanos a 0 indican mayor monodispersidad en las muestras, mientras que los valores cercanos a 1 indican mayor variabilidad en los tamaños (Lancheros et al., 2014). De acuerdo con los resultados obtenidos de PDI, todos los valores fueron inferiores a 0.5 , indicando que las nanoemulsiones resultantes se caracterizaban por tener una distribución de tamaño monomodal. De manera similar, Guttof et al. (2015) obtuvieron que al utilizar Tween 80 a una alta concentración (10\% p/p) es posible obtener un menor tamaño de partícula $(<200 \mathrm{~nm})$ y PDI $(<0.3)$ en nanoemulsiones con vitamina $\mathrm{D}$. Destacando que el tipo y concentración de 
Mundo Nano | ARTículos | www.mundonano.unam.mx

12(23), 1e-15e, julio-diciembre 2019 | http://dx.doi.org/10.22201/ceiich.24485691e.2019.23.67653

Mujica-Álvarez, J., Matiacevich, S. y Bustos, R.

TABLA 1. Tamaño promedio y distribución, y periodo de inducción (PI) para cada corrida experimental del diseño Box-Behnken.

\begin{tabular}{|c|c|c|c|c|c|c|c|c|}
\hline Corrida & $\begin{array}{c}\text { Tipo } \\
\text { encapsulante }\end{array}$ & $\begin{array}{c}\text { Tween } \\
80 \\
(\% \mathrm{p} / \mathrm{p}) \\
\end{array}$ & $\begin{array}{l}\text { Tiempo } \\
\text { (min) }\end{array}$ & $\begin{array}{l}\text { Tamaño } \\
(\mathrm{nm})\end{array}$ & PDI & $\begin{array}{l}\text { PI } \\
80 \\
{ }^{\circ} \mathrm{C} \\
\text { (h) } \\
\end{array}$ & $\begin{array}{c}\text { PI } \\
110 \\
{ }^{\circ} \mathrm{C} \\
\text { (h) } \\
\end{array}$ & $\begin{array}{c}\text { PI } \\
140 \\
{ }^{\circ} \mathrm{C} \\
\text { (h) } \\
\end{array}$ \\
\hline 1 & $\mathrm{CAP} / \mathrm{CS}$ & 1.5 & 2 & $23.7 \pm 0.8^{\mathrm{bc}}$ & $0.22 \pm 0.02$ & 10.2 & 3.2 & 2.1 \\
\hline 2 & CS & 1.5 & 1 & $42.4 \pm 0.1^{9}$ & $0.32 \pm 0.02$ & 10 & 4.2 & 2.8 \\
\hline 3 & CS & 2 & 2 & $19.6 \pm 0.2^{\mathrm{a}}$ & $0.230 \pm 0.001$ & 8.7 & 2.8 & 2.1 \\
\hline 4 & CS & 1 & 2 & $33.0 \pm 2.0^{\mathrm{e}}$ & $0.15 \pm 0.08$ & 12.2 & 4.5 & 3.7 \\
\hline 5 & CAP & 1.5 & 3 & $53.2 \pm 0.7^{i}$ & $0.13 \pm 0.08$ & 8.3 & 3.2 & 2 \\
\hline 6 & $\mathrm{CAP} / \mathrm{CS}$ & 2 & 1 & $35.9 \pm 1.3^{f}$ & $0.28 \pm 0.07$ & 15.1 & 3.5 & 3.6 \\
\hline 7 & CAP & 1 & 2 & $53.6 \pm 0.8^{i}$ & $0.21 \pm 0.04$ & 9.8 & 3 & 1.9 \\
\hline 8 & $\mathrm{CAP} / \mathrm{CS}$ & 2 & 3 & $18.2 \pm 0.4^{\mathrm{a}}$ & $0.231 \pm 0.002$ & 9.8 & 3.2 & 2.3 \\
\hline 9 & $\mathrm{CAP} / \mathrm{CS}$ & 1.5 & 2 & $26.8 \pm 0.1^{\mathrm{cd}}$ & $0.25 \pm 0.01$ & 9.8 & 2.9 & 2.1 \\
\hline 10 & $\mathrm{CS}$ & 1.5 & 3 & $23.1 \pm 0.5^{b}$ & $0.228 \pm 0.004$ & 12.2 & 2.6 & 3.3 \\
\hline 11 & CAP & 2 & 2 & $35.7 \pm 0.3^{f}$ & $0.25 \pm 0.02$ & 12 & 3.4 & 2.9 \\
\hline 12 & CAP & 1.5 & 1 & $49.1 \pm 0.7^{\mathrm{h}}$ & $0.30 \pm 0.07$ & 28.6 & 3.9 & 2.8 \\
\hline 13 & $\mathrm{CAP} / \mathrm{CS}$ & 1 & 3 & $71.8 \pm 1.7^{j}$ & $0.11 \pm 0.11$ & 14.9 & 6.5 & 4.5 \\
\hline 14 & CAP/CS & 1.5 & 2 & $29.1 \pm 0.1^{\text {de }}$ & $0.27 \pm 0.01$ & 11.1 & 2.5 & 2.9 \\
\hline 15 & $\mathrm{CAP} / \mathrm{CS}$ & 1 & 1 & $99.2 \pm 2.0^{k}$ & $0.16 \pm 0.16$ & 12.1 & 4.2 & 4.3 \\
\hline
\end{tabular}

Nota: ${ }^{a}, b, c, d, e, f, g, h, i, j, k$ Letras diferentes dentro de columna indican diferencias significativas a $\mathrm{P}<0.05$.

Fuente: Elaboración de los autores.

surfactante es importante en la obtención de un menor tamaño de partícula y PDI. Por otra parte, Cheong y Nyam (2016) realizaron nanoemulsiones de aceite de semillas de kenaf con caseinato de sodio y Tween 20, y señalaron que el aumento de la concentración de surfactante (5-10\% p/p) disminuyó la uniformidad de la distribución de tamaño de partícula. Sin embargo, sus valores de PDI fluctuaron entre 0.107 y 0.140 . indicando que estos valores correspondían de igual manera a una distribución de tamaño monodispersa a pesar del efecto observado.

De acuerdo con el diseño experimental, el Tween 80 tuvo un efecto estadísticamente significativo sobre el tamaño de nanopartícula, en donde a una mayor concentración de Tween 80 menor es el tamaño. Este efecto se atribuye a la presencia del tensioactivo en la interfase, lo cual facilita la formación de pequeñas gotas, reduciendo así el tamaño de las partículas finales (Martins et al., 2016; McClements, 2016). El Tween 80, al ser una molécula pequeña, es más eficaz que los polímeros en disminuir el diámetro de gota debido a su rápida adsorción en la superficie de la partícula (McClements, 2016).

Con respecto al índice de polidispersidad, se observó que el tiempo de ultrasonido tuvo un efecto negativo $(\mathrm{p}<0.005)$ sobre el PDI, es decir, a mayor tiempo de ultrasonido se incrementó este valor, siendo el objetivo tener un 
índice bajo para garantizar así una distribución homogénea de tamaño de nanopartículas. De manera contraria, la concentración de Tween 80 tuvo un efecto positivo ( $\mathrm{p}<0.005)$ sobre esta respuesta, es decir, a mayor concentración del tensoactivo se logró disminuir el PDI. El tipo y la concentración de tensioactivo no solo son importantes en la estabilización de emulsiones durante el proceso de nanoemulsificación, sino que también ayudan a prevenir la agregación de las gotas, manteniendo así un valor de PDI bajo. Sin embargo, la concentración de Tween 80 como efecto individual, tiene un comportamiento cuadrático ya que al utilizar concentraciones por sobre el $2 \%$ $\mathrm{p} / \mathrm{p}$, el valor de PDI aumenta. Esto debido a la formación de micelas que genera un exceso de Tween 80.

Por otra parte, el tiempo de ultrasonido tuvo un efecto significativo sobre el tamaño promedio de partícula y PDI: a medida que el tiempo aumentó, tanto el tamaño como el PDI disminuyeron. Esto se debe a que existe un aumento en las fuerzas de corte aplicadas sobre las gotas formadas, causándoles deformación y fragmentación y, en consecuencia, un menor tamaño (Shahavi et al., 2015).

\section{Análisis de estabilidad oxidativa de nanoemulsiones con vitamina $\mathrm{E}$}

Siendo el ultrasonido una metodología que puede afectar la estructura y funcionalidad de compuestos naturales como las vitaminas (Hosseini y Hosseini, 2017), es importante considerar que la vitamina E no se afecta por este tratamiento mientras sean utilizadas, como en este trabajo, bajas frecuencias $(20 \mathrm{MHz}$ ) y baja densidad de potencia <1000 W/L (Fernandes et al., 2016; Hosseini y Hosseini, 2017). Por otro lado, la utilización de ultrasonido ha sido previamente utilizado tanto para la extracción de vitamina E de fuentes naturales como el aceite de oliva (Nunes et al., 2018), así como para la caracterización de nanoemulsiones con base en vitamina E sin afectar sus propiedades (Cheong et al., 2008; Hosseini y Hosseini, 2017) o funcionalidad (Meghani et al., 2018). Es por ello que, en primer lugar, se evalúa su capacidad de oxidación tanto libre como en la nanoemulsión. La tabla 1 muestra el periodo de inducción (PI) medido a 80, 110 y $140{ }^{\circ} \mathrm{C}$ de cada corrida experimental de nanoemulsión con vitamina $\mathrm{E}$.

Se observó que a medida que aumenta la temperatura los valores de periodo de inducción disminuyen tanto de la vitamina libre como de la emulsionada. Esto se debe a la degradación que sufre la matriz encapsulante a una alta temperatura, quedando expuesta la vitamina, la cual es sensibles a factores como la temperatura y el oxígeno, lo cual se demuestra por el bajo PI de la vitamina libre. De la misma forma, las vitaminas son susceptibles a degradarse durante el procesamiento y almacenamiento, y a reaccionar con otros componentes del sistema alimentario (Sanguansri y Augustin, 2010).

Por otra parte, la interacción entre el tipo de agente encapsulante y el tiempo de ultrasonido tienen un efecto significativamente negativo para el periodo de inducción a $80^{\circ} \mathrm{C}$. El tipo de encapsulante para maximizar el pe- 
riodo de inducción es el Capsul ${ }^{\circledR}$, aunque el caseinato de sodio permite obtener un menor tamaño de nanocápsulas y proporciona un mayor contenido proteico en la elaboración final del ingrediente vitamínico encapsulado.

Para las temperaturas de 110 y $140{ }^{\circ} \mathrm{C}$ no hubo factores significativos. Esto se debe a que a altas temperaturas las matrices estudiadas pierden su capacidad encapsulante y/o protectora y se degradan causando que la vitamina quede expuesta a procesos oxidativos (Kiokias et al., 2017).

\section{Optimización de múltiples respuestas del diseño experimental Box-Behnken}

La optimización de múltiples respuestas permite determinar las configuraciones de los factores experimentales que cubren las características deseadas para una o más respuestas simultáneamente. Esto se hace mediante la construcción de la función de deseabilidad, basada sobre los valores de las variables de respuesta. La función se define dentro de un valor entre 0 y 1 . En la figura 1 se expresa gráficamente la superficie de respuesta, en donde para el modelo establecido el índice de deseabilidad fue de 0.72 con un error asociado del $28 \%$. Este modelo optimiza tres variables de respuesta diferentes, donde se minimiza el tamaño y PDI, y se maximizan los periodos de inducción a 80,110 y $140{ }^{\circ} \mathrm{C}$.

A partir del diseño experimental se determinó que las condiciones óptimas fueron: caseinato de sodio como agente encapsulante, $1 \% \mathrm{p} / \mathrm{p}$ de Tween 80 y 3 min de ultrasonido. En la tabla 2, se señalan los valores teóricos (o esperados) de las variables de respuestas a partir de los parámetros óptimos obtenidos y los valores reales correspondientes a la validación del modelo.

De acuerdo con estos resultados, el modelo fue validado a pesar de existir una diferencia entre el tamaño de partícula teórico y real, pues este último valor cumplía con lo definido como nanoemulsión, y, por otra parte,

FIGURA 1. Superficie de respuesta estimada para el efecto de la concentración de Tween 80, tipo de agente encapsulante y tiempo de ultrasonido sobre la optimización de múltiple respuesta.

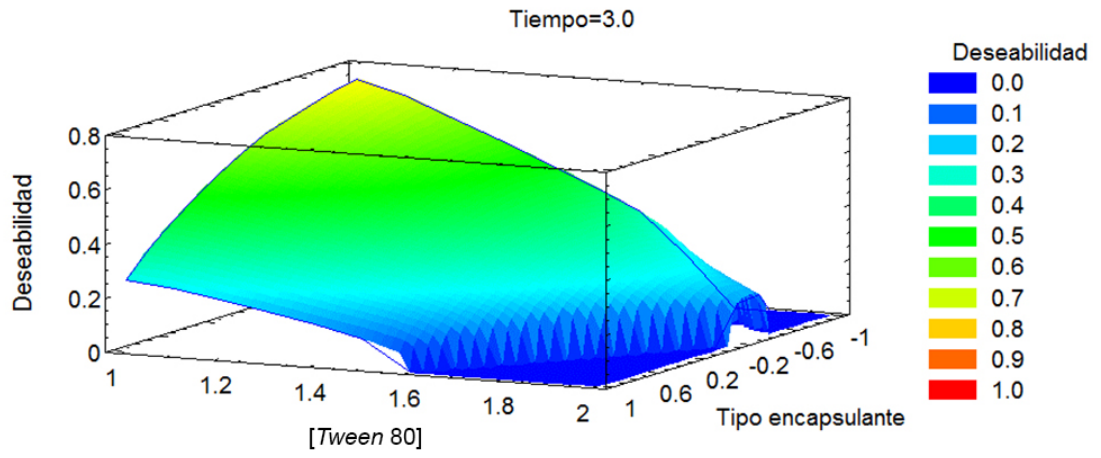

Fuente: Elaboración de los autores. 
TABLA 2. Valores teóricos y reales de las variables de respuesta a partir de las condiciones óptimas obtenidas según diseño experimental Box-Behnken.

\begin{tabular}{c|c|c} 
Factor & Valor teórico & Valor real \\
\hline Tamaño $(n m)$ & PI $140^{\circ} \mathrm{C}$ & $80 \pm 3^{\mathrm{b}}$ \\
\hline $\mathrm{PDI}$ & $0.14 \pm 0.03^{\mathrm{c}}$ & $0.18 \pm 0.06^{\mathrm{c}}$ \\
\hline $\mathrm{PI} 80^{\circ} \mathrm{C}$ & $12 \pm 4^{\mathrm{d}}$ & $8.7 \pm 0.4^{\mathrm{d}}$ \\
\hline $\mathrm{PI} 110^{\circ} \mathrm{C}$ & $5 \pm 1^{\mathrm{e}}$ & $4.1 \pm 0.8^{\mathrm{e}}$ \\
\hline $\mathrm{PI} 140^{\circ} \mathrm{C}$ & $5 \pm 1^{\mathrm{f}}$ & $2.6 \pm 0.1^{\mathrm{f}}$ \\
\hline
\end{tabular}

Nota: ${ }^{a, b, c, d, e, f}$ Letras diferentes entre filas indican diferencias significativas a $\mathrm{P}<0.05$.

Fuente: Elaboración de los autores.

no existió una diferencia significativa entre las respuestas teóricas y reales de PDI y PI a 80 y $110^{\circ} \mathrm{C}$.

\section{Estabilidad fisica de las nanoemulsiones obtenidas a partir de las condiciones experimentales óptimas}

Una vez validado el modelo, se midió la estabilidad física en el tiempo de la nanoemulsión obtenida analizando los perfiles de retrodispersión o backscattering (\%BS). La emulsión se almacenó a $4{ }^{\circ} \mathrm{C}$ durante $24 \mathrm{~h}$. Sin embargo, esta concentración solo se mantuvo estable durante 2 horas. Considerando que la nanoemulsión pasaría a una etapa de secado por liofilización, era necesario que la emulsión se mantuviese estable por un rango más amplio de tiempo. Para ello es importante considerar que la actividad emulsificante del caseinato de sodio puede modificarse por el proceso de ultrasonido, donde cambios en la conformación proteica aumentan la hidrofobicidad superficial, generando mayor actividad emulsificante de la proteína (de Figueiredo Furtado et al., 2017). Sin embargo, esto no necesariamente indica mayor estabilidad, ya que un exceso de la proteína genera inestabilidad por floculación debido a proteína no adsorbida (Dickinson y Golding, 1997a; Álvarez Cerimedo et al., 2010). Huck-Iriart et al. (2011a) reportaron también la importancia del ratio aceite:caseinato, donde un ratio de 2 mostró una larga estabilidad en emulsiones procesadas por ultrasonido. Este aumento en la estabilidad de las emulsiones se atribuyó al aumento tanto en la viscosidad de la fase continua como en la estabilidad de la interfase (Dickinson y Golding, 1997b). Por lo tanto, para obtener emulsiones estables, se debe considerar dicho parámetro, pero la estabilidad de la emulsión también depende de los componentes y de las condiciones de proceso (Álvarez Cerimedo et al., 2010; Huck-Iriart et al., 2011b). Por esta razón, considerando lo anteriormente expuesto, es que se realizaron pruebas aumentando la concentración del caseinato de sodio de $4 \%$ p/p a $8 \%$ p/p, obteniendo de esta forma un ratio aceite:caseinato de 1.5. La figura 2 muestra la estabilidad física de una nanoemulsión con $4 \% \mathrm{p} / \mathrm{p}$ y $8 \% \mathrm{p} / \mathrm{p}$ de caseinato de sodio, siendo esta última estable hasta $72 \mathrm{~h}$ almacenada a $4{ }^{\circ} \mathrm{C}$, sin observarse separación de fases. 
Mundo Nano | ARTículos | www.mundonano.unam.mx

12(23), 1e-15e, julio-diciembre 2019 | http://dx.doi.org/10.22201/ceiich.24485691e.2019.23.67653

Mujica-Álvarez, J., Matiacevich, S. y Bustos, R.

FIGURA 2. Perfil de retrodispersión o backscattering (\%BS) en función de la longitud del tubo: estabilidad física de nanoemulsión con vitamina $\mathrm{E}$ con $4 \% \mathrm{p} / \mathrm{p}$ (a) y $8 \% \mathrm{p} / \mathrm{p}$ (b) de caseinato de sodio.
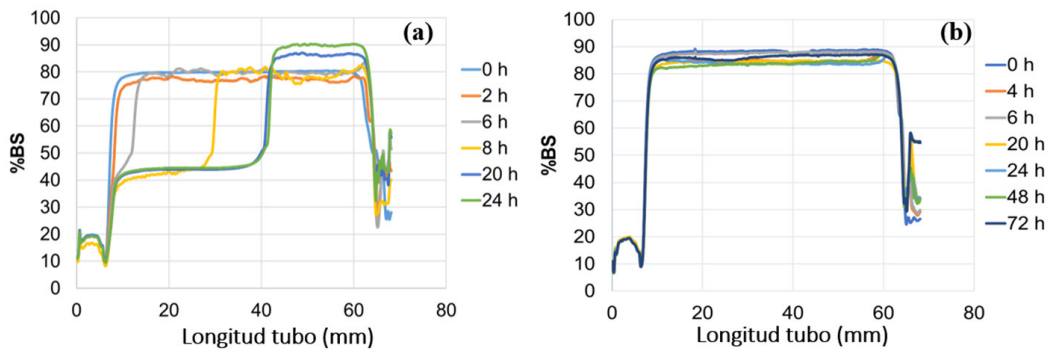

Fuente: Elaboración de los autores.

La desestabilización de la nanoemulsión con $4 \%$ p/p de CS se asocia principalmente con los fenómenos de coalescencia y/o cremado, provocando un aumento del tamaño de gotas, finalmente culmina con la separación completa de la fase acuosa y oleosa (Katouzian y Jafari, 2016; Mayer et al., 2013; Tadros, 2009). También, alrededor de $20 \mathrm{~h}$ de almacenamiento existe un aumento del porcentaje de retrodispersión, atribuido al fenómeno de cremado, en donde las gotas migran a la parte superior del tubo formando una capa de crema. La formación de cremado en las suspensiones se puede dar por diferentes razones, dependiendo de la concentración de las nanocápsulas, polidispersidad e interacción entre partículas (Robins, 2000).

Los nuevos valores de respuesta al aumentar a $8 \% \mathrm{p} / \mathrm{p}$ de CS se muestran en la tabla 3. Estos variaron con respecto a los obtenidos utilizando un $4 \% \mathrm{p} / \mathrm{p}$ de CS, sin embargo, los valores obtenidos fueron aceptables con base en el modelo estudiado.

Por último, la concentración se cambió de CS ( 4 a $8 \%$ p/p) y el resto de las variables, concentración de Tween 80 y tiempo de ultrasonido, no se modificaron. De esta forma se dio paso a la etapa de secado de las nanoemulsiones.

TABLA 3. Valores de respuestas de nanoemulsión con vitamina E con una concentración $8 \% \mathrm{p} / \mathrm{p}$ de caseinato de sodio.

\begin{tabular}{|c|c|}
\hline Respuesta & Valor óptimo \\
\hline Tamaño (nm) & $63 \pm 1$ \\
\hline PDI & $0.20 \pm 0.05$ \\
\hline $\mathrm{PI} 80^{\circ} \mathrm{C}$ & $10.2 \pm 0.4^{\mathrm{a}}$ \\
\hline $\mathrm{PI} 110^{\circ} \mathrm{C}$ & $4.4 \pm 0.4^{\mathrm{b}}$ \\
\hline $\mathrm{PI} 140^{\circ} \mathrm{C}$ & $3 \pm 1^{\mathrm{c}}$ \\
\hline
\end{tabular}

Nota: ${ }^{a}, \mathrm{~b}, \mathrm{c}$ Letras diferentes dentro de columna indican diferencias significativas a $\mathrm{P}<0.05$. Fuente: Elaboración de los autores. 


\section{Secado por liofilización de nanoemulsiones con vitamina $\mathrm{E}$}

La liofilización se utiliza ampliamente para mejorar la estabilidad durante el almacenamiento de diversos productos, entre ellos las nanoemulsiones (Khayata, 2012). En este caso, se midió la estabilidad oxidativa también de los polvos conteniendo vitamina E nanoencapsulada a temperaturas de 80 , 110 y $140^{\circ} \mathrm{C}$.

La tabla 4 detalla los valores de periodo de inducción en horas obtenidos para las distintas temperaturas. Se observó que la estabilidad oxidativa de los polvos liofilizados fue mayor que el de las nanoemulsiones y la vitamina E no encapsulada. Estos resultados están dentro de lo esperado ya que en el caso de la vitamina E no encapsulada, esta no contaba con la protección de un agente encapsulante. A partir de esto se observó que el PI del polvo liofilizado a $80^{\circ} \mathrm{C}$ es 7 veces mayor en comparación con la vitamina E libre y 2.5 veces más que la vitamina $\mathrm{E}$ encapsulada por nano-emulsificación. Esto se atribuye a que durante el proceso de liofilización se elimina el agua que eventualmente podría participar en procesos oxidativos.

Por otra parte, en la figura 3 se muestra el cambio de color de la nanoemulsión de vitamina E, polvo liofilizado y vitamina E libre. Se observa que a medida que aumenta la temperatura, existe una coloración marrón más intensa, lo cual implica un grado de oxidación mayor de la muestra.

TABLA 4. Valores de periodo de inducción en horas (h) de vitamina E libre, nanoemulsión con vitamina E y nanoemulsión liofilizada.

\begin{tabular}{c|c|c|c} 
Muestra & $80^{\circ} \mathrm{C}$ & $110^{\circ} \mathrm{C}$ & $140^{\circ} \mathrm{C}$ \\
\hline Vitamina E libre & $3.6 \pm 0.5^{\mathrm{a}}$ & $1.8 \pm 0.4^{\mathrm{d}}$ & $1.0 \pm 0.3^{\mathrm{f}}$ \\
\hline Nanoemulsión & $10.2 \pm 0.4^{\mathrm{b}}$ & $4.4 \pm 0.4^{\mathrm{e}}$ & $3.0 \pm 1.0^{\mathrm{g}}$ \\
\hline Liofilizado & $26.3 \pm 0.3^{\mathrm{c}}$ & $4.2 \pm 0.5^{\mathrm{e}}$ & $4.1 \pm 0.1^{\mathrm{s}}$ \\
\hline
\end{tabular}

Nota: $a, b, c, d, e, f, g$ Letras diferentes dentro de columna indican diferencias significativas a $\mathrm{P}<0.05$. Fuente: Elaboración de los autores.

FIGURA 3. Vitamina E libre, nanoemulsión de vitamina E y nanoemulsión liofilizada posterior al análisis de estabilidad oxidativa. Se incluye muestra control antes del análisis.

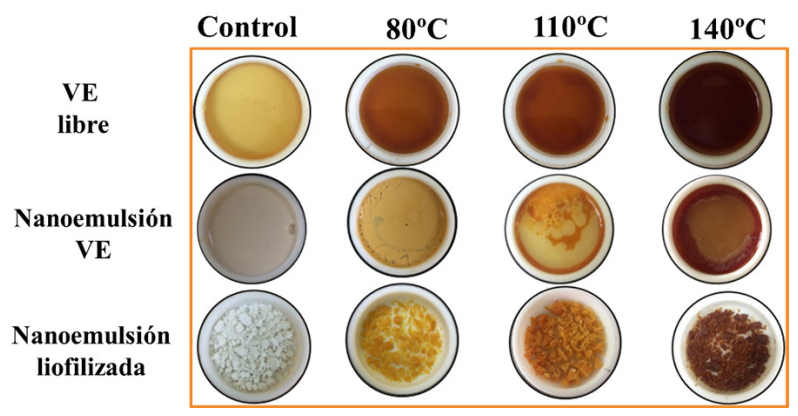

Fuente: Elaboración de los autores. 
De acuerdo con estos resultados, la tecnología de encapsulación permitió proteger una vitamina sensible a condiciones ambientales desfavorables, sin embargo, a temperaturas sobre los $110^{\circ} \mathrm{C}$ no existe una protección significativa del compuesto bioactivo, pues fue posible observar una mayor coloración marrón a rangos altos de temperatura, incluso en el producto liofilizado. Esto se refuerza con los valores de periodo de inducción obtenidos tanto para 110 y $140{ }^{\circ} \mathrm{C}$, en los cuales no existe una diferencia considerable para las tres muestras evaluadas.

\section{Conclusión}

La utilización de la nanoencapsulación en la industria alimentaria permite proteger e incorporar de mejor manera un compuesto bioactivo a un alimento ya que a escala nano mejora la estabilidad del compuesto encapsulado. De esta manera es posible obtener una liberación controlada y prolongada del mismo en un sitio específico porque se reduce la posibilidad de que el compuesto pueda interactuar con otras moléculas presentes en el medio, mejorando así su bioaccesibilidad y biodisponibilidad dentro del organismo. Es así como en este trabajo se logró exitosamente encapsular vitamina E utilizando como agente encapsulante caseinato de sodio mediante la técnica de emulsificación asistida con ultrasonido.

A partir de la optimización se logró encontrar las condiciones que permiten disminuir el tamaño de las nanopartículas e índice de polidispersidad, donde la concentración de Tween 80 y tiempo de ultrasonido tienen una importante función en la determinación de estos parámetros. Además, se mejoró la estabilidad física de la nanoemulsión con vitamina $\mathrm{E}$ aumentando la concentración del agente encapsulante a un $8 \% \mathrm{p} / \mathrm{p}$.

Por otra parte, los resultados de estabilidad oxidativa indicaron que el proceso de liofilización mejora significativamente la estabilidad de la vitamina $\mathrm{E}$ encapsulada en comparación con la nanoemulsión y la vitamina $\mathrm{E}$ libre, en donde el periodo de inducción del polvo a $80^{\circ} \mathrm{C}$ fue 7 veces mayor que la vitamina E libre y 2.5 veces mayor que la vitamina $\mathrm{E}$ encapsulada.

Proteger los compuestos bioactivos de altas temperaturas es muy importante, pues durante los procesos que comúnmente se realizan en la industria alimentaria podría verse afectada su actividad y, en consecuencia, inactivar sus propiedades funcionales tanto dentro del alimento como en el organismo.

Concluimos, de esta forma, que es posible obtener un ingrediente vitamínico nanoencapsulado, con buena estabilidad física y oxidativa, para ser incorporado en alimentos.

\section{Referencias}

Álvarez Cerimedo, M. S., Huck-Iriart, C., Candal, R. J., Herrera, M. L. (2010). Stability of emulsions formulated with high concentrations of sodium caseinate and 
trehalose. Food Research International, 43: 1482-1493.

http://dx.doi.org/10.1016/j.foodres.2010.04.008

Cheong, A. M., Nyam, K. L. (2016). Improvement of physical stability of kenaf seed oil-in-water nanoemulsions by addition of $ß$-cyclodextrin to primary emulsion containing sodium caseinate and Tween 20. Journal of Food Engineering, 183: 24-31. http://dx.doi.org/10.1016/j.jfoodeng.2016.03.012

Cheong, J. N., Tan, C. P., Che Man, Y. B., Misran, M. (2008). $\alpha$-Tocopherol nanodispersions: Preparation, characterization and stability evaluation. Journal of Food Engineering, 89(2): 204-209.

Dasgupta, N., Ranjan, S. (2018). An introduction to food grade nanoemulsions. Singapore: Springer.

Dickinson, E., Golding, M. (1997a). Depletion flocculation of emulsions containing unadsorbed sodium caseinate. Food Hydrocolloids, 11: 13-18.

http://dx.doi.org/10.1016/S0268-005X (97) 80005-7

Dickinson, E., Golding, M. (1997b). Rheology of sodium caseinate stabilized oil-inwater emulsions. Journal of Colloidal and Interface Science, 191: 166-176.

http://dx.doi.org/10.1006/jcis.1997.4939

De Figueiredo Furtado, G., Araújo Mantovani, R., Consoli, L., Dupas Hubinger, M., Lopesda Cunha, R. (2017). Structural and emulsifying properties of sodium caseinate and lactoferrin influenced by ultrasound process. Food Hydrocolloids, 63: 178-188. http://dx.doi.org/10.1016/j.foodhyd.2016.08.038

Fernandes, F. A. N, Oliveira, V. S., Gomes, W. F., Rodrigues, S. (2016). Degradation kinetics of vitamin E during ultrasound application and the adjustment in avocado purée by tocopherol acetate addition. LWT-Food Science and Technology, 69: 342-347.

Ghani, A. A., Adachi, S., Shiga, H., Neoh, T. L., Adachi, S., Yoshii, H. (2017). Effect of different dextrose equivalents of maltodextrin on oxidation stability in encapsulated fish oil by spray drying. Bioscience Biotechnology \& Biochemistry, 81: 705-711. http://dx.doi.org/10.1080/09168451.2017.1281721

Gómez-Cruz, N. I., Jiménez-Munguía, M. T. (2014). Métodos de secado de emulsiones alimentarias. Temas Selectos de Ingeniería de Alimentos, 8(2): 23-33.

Guttof, M., Saberi, A. H., McClements, D. J. (2015). Formation of vitamin D nanoemulsion-based delivery systems by spontaneous emulsification: factors affecting particle size and stability. Food Chemistry, 171: 117-122.

http://dx.doi.org/10.1016/j.foodchem.2014.08.087

Hosseini, S., Hosseini, S. (2017). Impact of process parameters in the generation of nanoemulsions containing omega 3 fatty acids and $\alpha$-tocopherol. International Journal of Nano Dimension, 8: 351-360.

Huck-Iriart, C., Candal., R. J., Herrera, M. L. (2011a). Effect of processing conditions and composition on sodium caseinate emulsions stability. Procedia Food Science, 1: 116-122.

Huck-Iriart, C., Álvarez-Cerimedo, M. S., Candal, R. J., Herrera, M. L. (2011b). Structures and stability of lipid emulsions formulated with sodium caseinate. Current Opinion in Colloid \& Interface Science, 16: 412-420. 
Katouzian, I., Jafari, S. M. (2016). Nano-encapsulation as a promising approach for targeted delivery and controlled release of vitamins. Trends in Food Science \& Technology, 53: 34-48. http://dx.doi.org/10.1016/j.tifs.2016.05.002

Khayata, N. (2012). Stability study and lyophilization of vitamin E-loaded nanocapsules prepared by membrane contactor. International Journal of Pharmaceutics, 439 (1-2): 254-59. https//dx.doi.org/10.1016/j.ijpharm.2012.09.032

Kiokias, S., Gordon M. H., Oreopoulou, V. (2017). Effects of composition and processing variables on the oxidative stability of protein-based and oil-in-water food emulsions. Critical Reviews in Food Science and Nutrition, 57(3): 549-558. http://dx.doi.org/10.1080/10408398.2014.893503

Lancheros, R. J., Beleño, J. A., Guerrero, C. A., Godoy-Silva, R. D. (2014). Producción de nanopartículas de PLGA por el método de emulsión y evaporación para encapsular N-Acetilcisteína (NAC). Universitas Scientiarum 19(C2): 161-168. http://dx.doi.org/10.11144/Javeriana.SC19-2.pnpm

Leong, T., Martin, G., Ashokkumar, M. (2016). Ultrasonic encapsulation - A review. Ultrasonics Sonochemistry, 35, Part B: 605-614. http://dx.doi.org/10.1016/j.ultsonch.2016.03.017

Martins, M., Loureiro, A., Azoia, N., Silva, C., Cavaco-Paulo, A. (2016). Protein formulations for emulsions and solid-in-oil dispersions. Trends in Biotechnology, 34(6): 496-505. http://dx.doi.org/1016/j.tibtech.2016.03.001

Mayer, S., Weiss, J., McClements, D. J., (2013). Vitamin E-enriched nanoemulsions formed by emulsion phase inversion: Factors influencing droplet size and stability. Journal of Colloid Interface Science, 402: 122-130. http://dx.doi.org/10.1016/j.jcis.2013.04.016

McClements, D. (2016). Food emulsions: Principles, practice and techniques. Estados Unidos: Editorial CRC Press.

Meghani, N., Patel, P., Kansara, K., Ranjan, S., Dasgupta, N., Ramalingam, C., Kumar, A. (2018). Formulation of vitamin D encapsulated cinnamon oil nanoemulsion: its potential anti-cancerous activity in human alveolar carcinoma cells. Colloids and Surfaces B: Biointerfaces, 166: 349-357. http://dx.doi.org/10.1016/j.colsurfb.2018.03.041

NIH (National Institute of Health) - US National Library of Medicine. (2018). Medline Plus. https://medlineplus.gov/ency/article/002400.htm

Nireesha, G. R., Divya, L., Sowmya, C., Venkateshan, N., Niranjan Babu, M., Lavakumar, V. (2013). Lyophilization/Freeze Drying - A Review. International Journal of Novel Trends in Pharmaceutical Sciences, 3(4): 87-98.

Nunes, A. M., Costa, A. S. G., Bessada, S., Santos, J., Puga, H., Alves, R. C., Freitas, V., Oliveira, M. B. P. P. (2018). Olive pomace as a valuable source of bioactive compounds: a study regarding its lipid- and water-soluble components. Science of the TotalEnvironment,644:229-236.http://dx.doi.org/10.1016/j.scitotenv.2018.06.350

Quirós-Sauceda, P., Ayala-Zavala, J. F., Olivas, G., González-Aguilar, G. (2014). Edible coatings as encapsulating matrices for bioactive compounds: a review. Journal of Food Science and Technology 51(9): 1674-1685.

http://dx.doi.org/10.1007/s13197-013-1246-x 
Robins, M. (2000). Emulsions - creaming phenomena. Current opinion in Colloid and Interface Science, 5(5-6): 265-272.

Rubio-Anaya, M., Guerrero-Beltrán, J. (2012). Polímeros utilizados para la elaboración de películas biodegradables. Temas Selectos de Ingeniería de Alimentos 6-2: 173-181.

Sanguansri, L, Augustin, M. A. (2010). Functional food product development. Canadá: Wiley-Blackwell.

Shahavi, M. H., Hosseini, M., Jahanshahi, M., Meyer, R. L., Darzi, G. N. (2015). Evaluation of critical parameters for preparation of stable clove oil nanoemulsion. Arabian Journal of Chemistry, 122:313-320.

http://dx.doi.org/10.1016/j.arabjc.2015.08.024

Tadros, T. (2009). Emulsion science and technology: a general introduction. Alemania: Wiley-VCH Verlag GmbH and Co.

Vidya, D., Shubhangi, S., Magdum, C., Mohite, S., Nitalikar, M. (2015). A review: dry emulsion. Asian Journal of Pharmaceutical Research, 5(4): 208-210. 